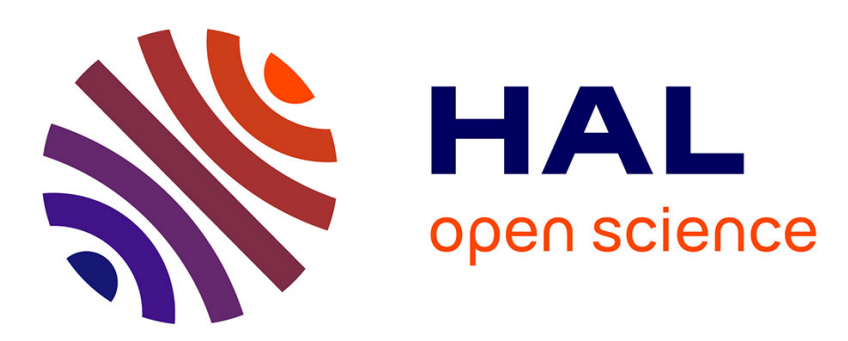

\title{
Reactive synthesizing of human locomotion combining nonholonomic and holonomic behaviors
}

\author{
Tan-Viet-Anh Truong, David Flavigne, Julien Pettré, Katja Mombaur, \\ Jean-Paul Laumond
}

\section{> To cite this version:}

Tan-Viet-Anh Truong, David Flavigne, Julien Pettré, Katja Mombaur, Jean-Paul Laumond. Reactive synthesizing of human locomotion combining nonholonomic and holonomic behaviors. IEEE/RASEMBS International Conference on Biomedical Robotics and Biomechatronics (BIOROB 2010), Sep 2010, Tokyo, Japan. inria-00539220

\section{HAL Id: inria-00539220 \\ https://inria.hal.science/inria-00539220}

Submitted on 24 Nov 2010

HAL is a multi-disciplinary open access archive for the deposit and dissemination of scientific research documents, whether they are published or not. The documents may come from teaching and research institutions in France or abroad, or from public or private research centers.
L'archive ouverte pluridisciplinaire HAL, est destinée au dépôt et à la diffusion de documents scientifiques de niveau recherche, publiés ou non, émanant des établissements d'enseignement et de recherche français ou étrangers, des laboratoires publics ou privés. 


\title{
Reactive synthesizing of human locomotion combining nonholonomic and holonomic behaviors
}

\author{
Tan-Viet-Anh Truong*, David Flavigne*, Julien Pettré**, Katja Mombaur*, Jean-Paul Laumond* \\ *LAAS-CNRS, University of Toulouse \\ 7 Avenue du Colonel Roche, 31077 Toulouse, France \\ \{atruong, dflavign, kmombaur, jpl\}@laas.fr \\ **Bunraku team, IRISA / INRIA-Rennes, France \\ julien.pettre@irisa.fr
}

\begin{abstract}
The trajectories of walking humans can be divided into two major classes: first, nonholonomic trajectories during which the orientation of the human body and the one of the motion are aligned (e.g., straight walking), and second, holonomic ones during which lateral velocities are observed (e.g., side-steps). The major contribution of this work is a general locomotion synthesis method for digital actors which enables combining nonholonomic and holonomic walk behaviors. Our motion synthesis technique is based on a motion capture blending method. Such a method is able to transform a low-dimensional trajectory describing the global displacement of a digital actor into a high-dimensional motion which involves all the degrees of freedom of the digital actor body. We extend previously existing motion blending techniques [12] to reach this goal. More precisely, we consider lateral velocity as a new input of the motion synthesis problem, whereas only tangential and angular were initially considered. Thus, the digital actor locomotion is controlled from 3 inputs in our approach: the tangential velocity, the angular velocity and the lateral velocity. The paper describes the major steps of our approach. At pre-processing, we analyze some motion captured locomotion cycles to build a Motion Library. The content of the library is projected into the Control Space. Then, at runtime, input (i.e., desired walk velocities) is also projected into the Control Space. We deduce a selection of motion captured locomotion cycles from the library, which are finally interpolated to generate a locomotion animation for digital actors. The newly generated locomotion cycle exhibit the desired input velocities.
\end{abstract}

\section{INTRODUCTION}

Human locomotion has been widely studied in various disciplines. Neuroscientists carried out research on the multisensory aspects (visual, vestibular inputs, etc) of the human walking [3], [4], and on the human nervous system control strategy [6], [5]. Biomechanics provided detailed descriptions of the human gait [14], [1]. Roboticists focused their attention on controlling walking machines, ranging from simple passive biped robots to more complex humanoid robots. Major issues are to maintain balance for these redundant systems during walking (as pioneered in [13]), to generate locomotion patterns (e.g., by using ZMP theory [17]), and to solve the inverse kinematics/dynamics problem for controlling the whole body motion [8].

It is known that humans exhibit trajectories where the body orientation supports the tangent direction of motion. In neuroscience, this statement is showed by the stereotypical nature of walking paths [7]: human always achieve similar global trajectories when reaching a given goal. In robotics, it is deduced from a well-known terminology for mobile robots motions: a nonholonomic constraint [9] integrated in

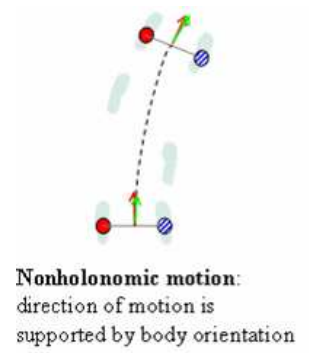

(a)

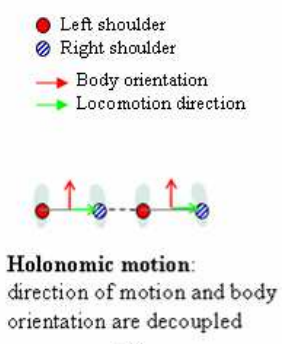

(b)
Fig. 1. The relationship between body orientation and direction of motion during human locomotion (cited from [15]).

the equation system:

$$
\left(\begin{array}{c}
\dot{x} \\
\dot{y} \\
\dot{\theta}
\end{array}\right)=\left(\begin{array}{c}
\cos \theta \\
\sin \theta \\
0
\end{array}\right) v_{f}+\left(\begin{array}{l}
0 \\
0 \\
1
\end{array}\right) \omega
$$

where $(x, y, \theta)$ is position and orientation of reference frame (the mid-point of two shoulders in our case). Nevertheless, in very specific situations, when targets are just nearby from the departure point, sideward or diagonal steps are performed [15] (see figure 1 (b)), and they are types of holonomic motions. In these cases, the above hypothesis of nonholonomic nature is not any longer true, and the equation (1) is not either validated. The holonomic factor (that makes us move sidewards) should be added. The equation system (1) is replaced then by another model (2) unifying nonholonomic and holonomic movements (presented in [10]).

$$
\left(\begin{array}{l}
\dot{x} \\
\dot{y} \\
\dot{\theta}
\end{array}\right)=\left(\begin{array}{c}
\cos \theta \\
\sin \theta \\
0
\end{array}\right) v_{f}+\left(\begin{array}{l}
0 \\
0 \\
1
\end{array}\right) \omega+\left(\begin{array}{c}
-\sin \theta \\
\cos \theta \\
0
\end{array}\right) v_{l}
$$

With this model, any configuration $(x, y, \theta)$ can be reached by angular $\omega$, forward $v_{f}$ and lateral $v_{l}$ (for sideward motions) velocity in a natural (human) way.

The problem is how to take into account the appearance of lateral velocities in global trajectories for deducing a whole body movement?

In this paper, we propose to generate animations of locomotion based on a motion capture blending technique. The locomotion controller is composed of three main components: the Motion library, the Control space and the Motion blending technique. Noting that we use tangential velocity instead of 


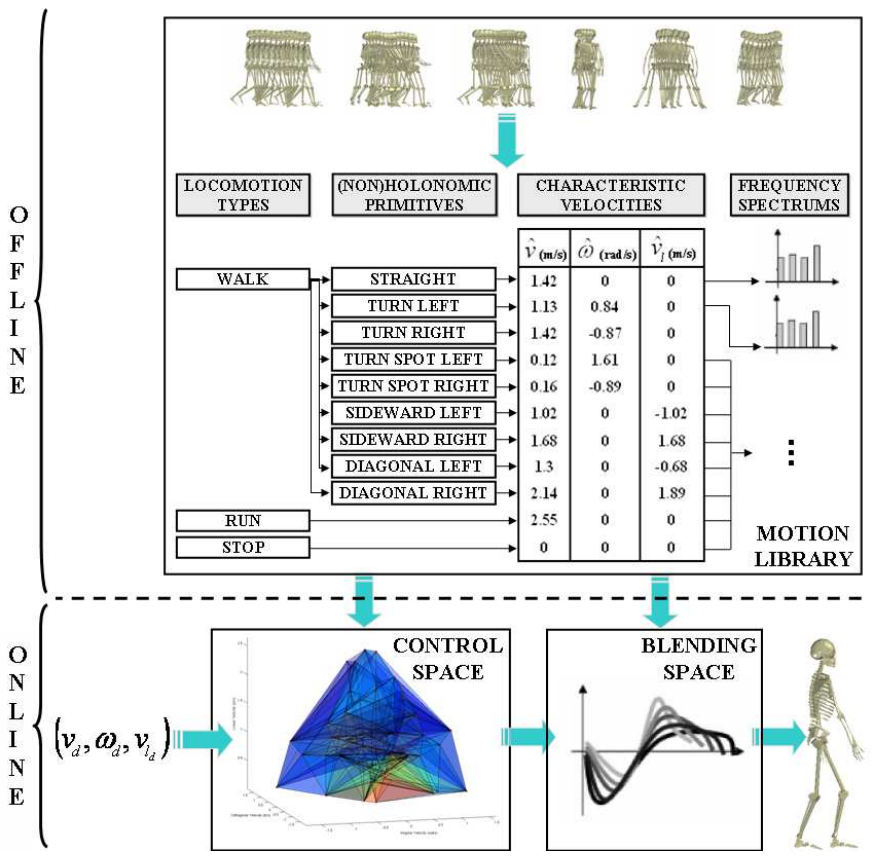

Fig. 2. Architecture of the locomotion controller. (To be adapted from [12]).

forward velocity because it is more intuitive for users to control directly the (total) velocity of human locomotion than trying to imply the forward velocity. So, the input of the controller is 3-dimensional: the desired tangential, angular and lateral velocities. The Control Space is the core of our method. This space is also 3-dimensional and structures the content of the Motion Library. A desired speed is given as input and is projected into velocity points cloud in 3D control space. This allows us to select the nearest neighbors (motion samples) and to find weights for each of them. Finally, the angular trajectories are expressed in the frequency domain using Fourier expansion (like in [16]) and interpolated.

The contribution of this paper is to extend the locomotion controller presented in [12] from 2 inputs to 3 input velocities by taking into account a desired lateral velocity.

The locomotion controller enables a human figure to follow predefined paths; the controller can thus be used in the context of locomotion planning for solving high-level goal reaching problems. We show examples of applications in the Results section. We also use the method proposed in [11] to obtain global trajectories between two desired configurations based on an inverse optimal control approach.

\section{Structure of the locomotion controller:}

Our locomotion controller includes two main processes: Offline building of the Motion Library and On-line full-body motion synthesis (see figure 2 for the global architecture of our method).

Off-line, we construct a Motion library which contains various human locomotions recorded from motion capture system. Motion captures are carefully recorded to satisfy our needs. Locomotion cycles are extracted from motion captures. Average velocities (tangential, angular and lateral) are ex- tracted for each cycle (respectively denoted as $\left.\left(\hat{v}, \hat{w}, \hat{v}_{l}\right)\right)$. The Motion Library building process is presented in section II.

The On-line animation process consists of interpolating cycles from library to synthesize movements with desired instantaneous velocities. This On-line process is composed of two components: Control space and Blending space. Given an input, cycles are selected from the library based on their characteristic average velocities. The Control Space structures the content of the library to enable this selection step: each locomotions cycle is projected as a $3 \mathrm{D}$ point into the control space, their coordinates are the average velocities. The resulting points cloud is structured using a 3D Delaunay triangulation. Given input $\left(v_{d}, w_{d}, v_{l_{d}}\right)$ is also projected into the control space. At each instant of animation, the input point is contained in a tetrahedron whose four vertices map to four locomotion samples (neighbors). These neighbors are finally interpolated. Angular trajectories of each degree of freedom are expressed as Fourier expansions to enable such an interpolation (Blending space). A new locomotion cycle with desired velocity features is synthesized.

Control space and Blending space are presented in section III and section IV respectively. Finally, some examples of animated locomtions are showed in the section $\mathrm{V}$.

\section{MOTION LIBRARY - MOTION CAPTURE DATABASE}

\section{A. Protocol and apparatus}

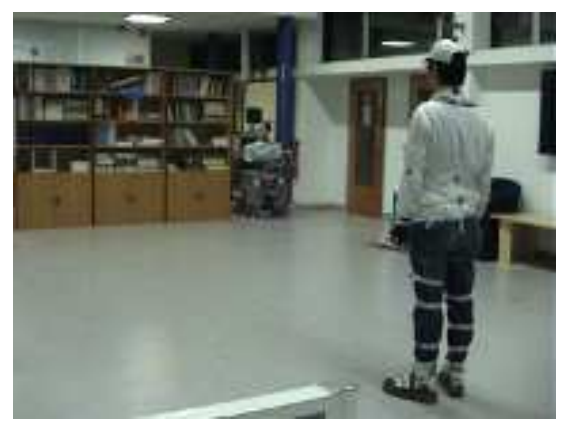

Fig. 3. The subject with markers in motion capture hall using for recording locomotions database.

We used the MotionAnalysis (from Motion Analysis Corporation, USA) motion capture system to record all locomotions of the Motion library. This system (equipped by LAAS-CNRS) wired to 10 cameras at a $100 \mathrm{~Hz}$ sampling frequency in the motion capture area $(4 \times 6(\mathrm{~m}))$ (figure 3 ). A single subject was equipped with 38 light reflective markers located on his whole body (see figure 6 (a)). These markers were attached on his head, neck, shoulders, spine, pelvis, legs and arms in order to acquire principal joint motions during walking. The subject was asked to perform all trials with a constant speed and in a natural way (like daily human walking).

Our purpose is to collect human locomotions with various velocities. These velocities are inferred from 3 basic locomotions: walk on a straight line, turn on the spot and step sidewards.

The two first types of locomotion represent nonholonomic behaviors, they are actually the forward walking on curved trajectories. We observed that by changing the curvature of 


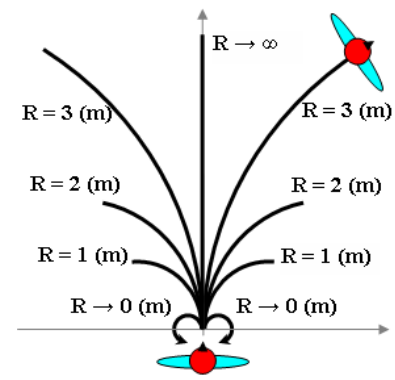

(a)

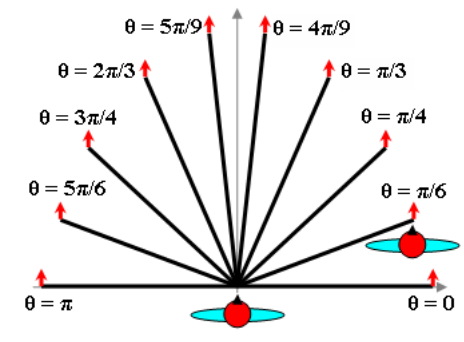

(b)
Fig. 4. The reachable space of locomotions in the library. (a) Nonholonomic locomotions sampled by curvature from radius 0 (turn on spot) to radius $1(\mathrm{~m})$, $2(\mathrm{~m}), 3(\mathrm{~m})$ (turn on the left, right) and infinitive radius (walk on a straight line). (b) Holonomic locomotions sampled by tangent direction $\theta$ of trajectory from initial to final configuration. This tangent direction $\theta$ represents sideward steps $(\theta$ are 0 and $\pi$ ) and diagonal steps $(\theta$ are from $\pi / 3$ to $5 \pi / 6)$.

the followed path, the three characteristic velocities change either. The curvature for straight walking is infinitive and corresponds to positive tangential velocity, whereas the angular and the lateral ones are null. The curvature for turning on the spot converges to zero and corresponds to positive or negative angular velocity only. Therefore, we sampled nonholonomic locomotions along curvatures from zero to infinitive on the left and the right to obtain the variance of tangential and angular velocities (see figure 4 (a)). Some examples of these recorded locomotions are shown in figure 5 (a).
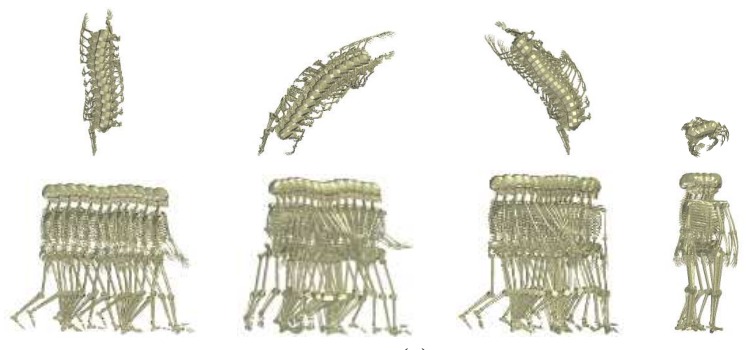

(a)
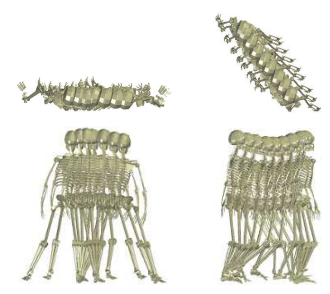

(b)

Fig. 5. Top view (on the top line) and side view (on the bottom line) of some nonholonomic locomotions (a) and holonomic locomotions (b) in the library. (a) From the left to the right of the figure, they are respectively Walking on a straight line, Turning on the right, Turning on the left and Turning on the spot. (b) Sideward step is on the left and Diagonal step is on the right of the figure.

The sideward walking is an holonomic type of locomotion. In this case, the tangential direction of trajectories are considered. This means that the variance of the tangential direction causes the variance of other velocities. For example, a diagonal walking and a sideward walking have different velocities. As a result, we sampled holonomic locomotions from zero to $\pi$ to obtain the variance of lateral and tangential velocities (see figure 4 (b)). Some examples of these recorded locomotions are shown in figure 5 (b).

For each of above types of locomotion, we recorded with 3 levels of tangential velocity: low, normal and fast. Straight running and rest (stop) postures were also captured for answering the needs of various scenarios. The subject performed 68 captures (68 locomotion primitives) total to compose the Motion library.

\section{B. Data analysis}

38 markers were tracked. Motion data were recorded as independent markers trajectories (points cloud) using the Cortex software from MotionAnalysis. The cartesian motions of these markers were used to calculate joint angles by using the module Calcium Solver. This application of Cortex uses a Global Optimization method for calculating the best fit of the markers data to the underlying fixed bone skeleton (figure 6 (a) to figure 6 (b)). All joint angles were then transferred into the avatar Eugene (figure 6 (c)) of Kineo Path Planner Interface (from Kineo CAM). Results were obtained by animating Eugene based on this interface.

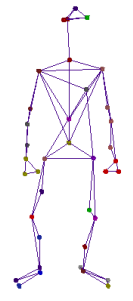

(a)

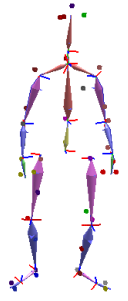

(b)

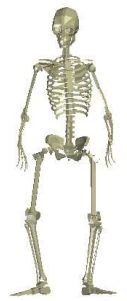

(c)
Fig. 6. The transfer from the subject to the avatar.

The block at the top of the figure 2 showed stages of the Motion library. After having recorded data we extracted one cycle from each entire locomotion sample. All locomotion cycles of the motion library have the same posture (the left toes push off) at starting position.

Then, we computed analytically the geometrical pattern corresponding to each locomotion sample in its trimmed cycle. The geometrical pattern is a line segment for locomotions on a straight line (including straight walking and sideward, diagonal steps). And it is an arc of circle for curved locomotions including turning left/right/on the spot. Three velocities of each capture were calculated in its corresponding trimmed cycle via the mid-point of two shoulders (it is the reference point considered in our current research in human locomotion [15], [11], and [10]). The discrete XYZ data of this point was used to calculate the tangential velocity $\hat{v}$ and the tangential direction $\theta_{T}$ of trajectory (figure 7). Orthogonal direction of shoulders is determined as :

$$
\theta_{S}=\theta_{l r}+\pi / 2
$$

where $\theta_{l r}$ is direction vector from left shoulder to right shoulder. Angular velocity is derived from $\theta_{S}$ and the lateral velocity is calculated with respect to $\hat{v}$ :

$$
\hat{v}_{l}=\hat{v} \sin \left(\theta_{S}-\theta_{T}\right)
$$




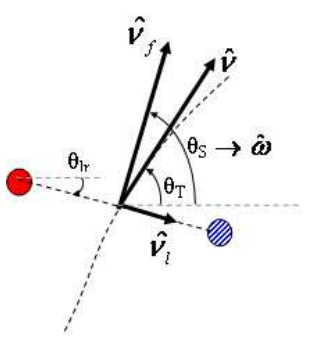

Fig. 7. Velocity components in a trajectory.

Some values of these velocities are presented in the Motion Library block of the figure 2 .

Because the motion of most degrees of freedom (head, neck, spine, arms, legs) are periodic and smooth, they are encoded in the frequency domain using a Discrete Fourier Transform. We computed the coefficient pair $\left(\alpha_{i}, \beta_{i}\right)$ corresponding to real and imaginary parts of Fourier series expansions. A motion of a degree of freedom is written as:

$$
m(t)=\frac{1}{2} \alpha_{0}+\sum_{k=1}^{N} \alpha_{k} \cos \left(\frac{k \pi t}{T}\right)+\beta_{k} \sin \left(\frac{k \pi t}{T}\right)
$$

where $N$ is the number of samples of transform and $T$ is the trimmed cycle of capture.

\section{3D CONTROL SPACE}

\section{A. Filling the control space}

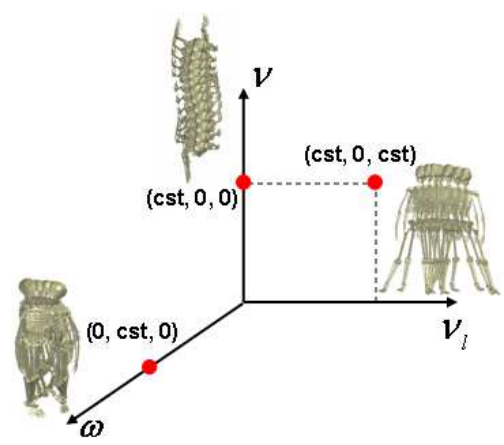

Fig. 8. 3D control space with three axis corresponding to three velocities. Three red points represent three basic locomotions. Their velocities set $\left(\hat{v}, \hat{\omega}, \hat{v}_{l}\right)$ are respectively $(c s t, 0,0),(0, c s t, 0)$ and $(c s t, 0, c s t)$.

This control space is based on 3 components: tangential, angular and lateral velocities. Each point in this control space is a set of $\left(v, \omega, v_{l}\right)$ calculated in the Motion library stage. The space was filled by motion samples stored in motion library. With 68 locomotions recorded, we had a data set of 68 points $\left(\hat{v}, \hat{\omega}, \hat{v}_{l}\right)$. Figure 8 shows three basic locomotions corresponding to three points in the space. They are: (1) walk on straight line with constant tangential velocity, (2) turning on the spot with constant angular velocity, and (3) stepping sideward with constant lateral and tangential velocities.

\section{B. Selecting locomotion samples}

Once an input is given in form of a set $\left(v_{d}, \omega_{d}, v_{l_{d}}\right)$, we have to find motions (neighbors) in library whose characteristic velocities are nearest to it. To do that, data points in the control space have to be structured into a 3D Delaunay triangulation. Figure 9 shows a tetrahedron in 3D Delaunay triangulation data structure composed of velocity points cloud in motion library. The input point is found in some tetrahedron. Four vertices of the found tetrahedron represent four locomotion samples used to synthesize the desired locomotion (see figure 10 for an illustration). By using CGAL (Computational Geometry Algorithms Library) [18], we can always find a tetrahedron containing the input point. The input point has to be in the envelope of the tetrahedra data structure.

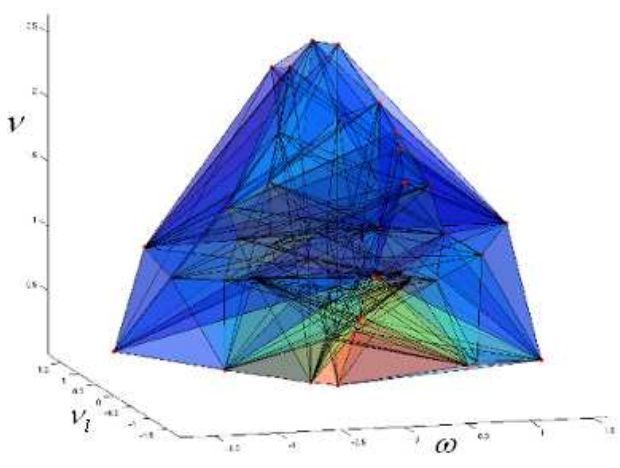

(a)

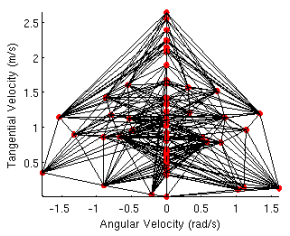

(b)

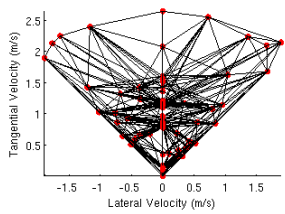

(c)
Fig. 9. The control space in from perspective view (a) and its projection into $(\omega, v)$ plan (b) and $\left(v_{l}, v\right)$ plan (c). Red points are vertices of tetrahedra structured by 3D Delaunay triangulation.

\section{Finding weights for selected samples tetrahedron}

Four locomotion samples of the found tetrahedron are used to calculate weights to be blended. Among these samples, the sample which velocities characteristics is nearest to $\left(v_{d}, \omega_{d}, v_{l_{d}}\right)$ has the greatest influence (weights) on the

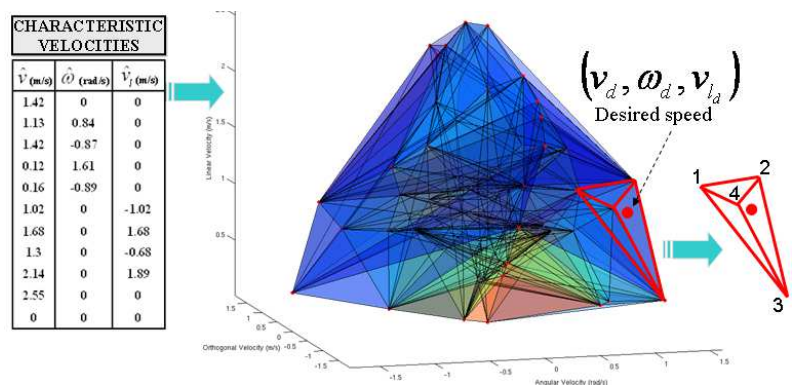

Fig. 10. Tetrahedron selection process from an input (desired speed). The red tetrahedron is selected from the tetrahedra data structure in the control space. 
result and vice versa. Weights are calculated by solving below equation system (as an interpolation in Finite Element Method [2]):

$$
\left\{\begin{array}{rrrrrr}
a \hat{v}_{1}+b \hat{v}_{2} & +c \hat{v}_{3}+d \hat{v}_{4}= & =v_{d} \\
a \hat{\omega}_{1}+b \hat{\omega}_{2} & +c \hat{\omega}_{3}+d \hat{\omega}_{4} & = & \omega_{d} \\
a \hat{v}_{l_{1}}+b \hat{v}_{l_{2}}+c \hat{v}_{l_{3}}+d \hat{v}_{l_{4}} & = & v_{l_{d}} \\
a+b & +c+d & = & 1
\end{array}\right.
$$

where $\left(\hat{v}_{1}, \hat{\omega}_{1}, \hat{v}_{l_{1}}\right),\left(\hat{v}_{2}, \hat{\omega}_{2}, \hat{v}_{l_{2}}\right),\left(\hat{v}_{3}, \hat{\omega}_{3}, \hat{v}_{l_{3}}\right),\left(\hat{v}_{4}, \hat{\omega}_{4}, \hat{v}_{l_{4}}\right)$ is respectively the tangential, angular and lateral velocities set of four selected locomotions (four vertices of found tetrahedron as in figure 10). Weights $(a, b, c, d)$ for these four samples are always in $[0,1]$ because input points are in the envelop of the $3 \mathrm{D}$ triangulation data structure. When an input is outside of the envelop, users should change the velocities directive.

\section{Motion BLENDING}

This stage consists of synthesizing a new locomotion from the found weights $(a, b, c, d)$ in the previous stage (see figure 11 for the process of the Motion blending). We first interpolate

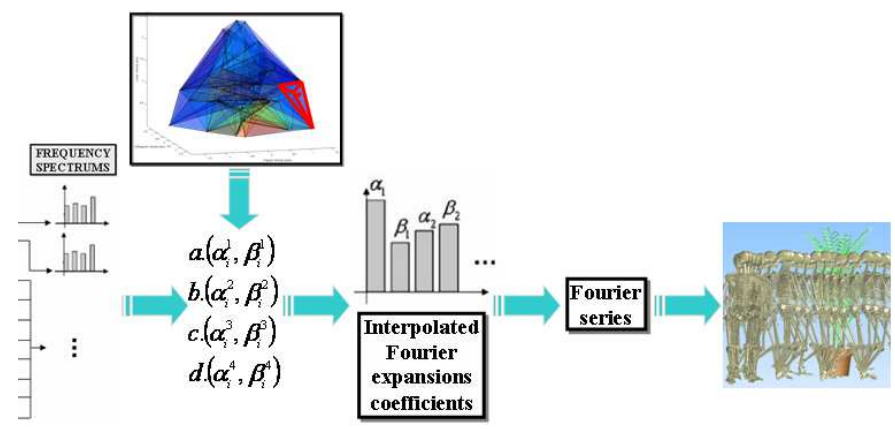

Fig. 11. Stages of the Motion blending.

the Fourier expansions of the locomotions corresponding to the four vertices of the tetrahedron:

$$
\left\{\begin{array}{c}
\alpha_{i}^{d}=a \alpha_{i}^{1}+b \alpha_{i}^{2}+c \alpha_{i}^{3}+d \alpha_{i}^{4} \\
\beta_{i}^{d}=a \beta_{i}^{1}+b \beta_{i}^{2}+c \beta_{i}^{3}+d \beta_{i}^{4}
\end{array}\right.
$$

where $\left(\alpha_{i}^{d}, \beta_{i}^{d}\right)$ and $\left(\alpha_{i}^{j}, \beta_{i}^{j}\right)$ are coefficients pair of joint angles of the desired locomotion and locomotion sample $\mathrm{j}$ respectively $(i=0 . . N)$. Then $\left(\alpha_{i}^{1}, \beta_{i}^{1}\right),\left(\alpha_{i}^{2}, \beta_{i}^{2}\right),\left(\alpha_{i}^{3}, \beta_{i}^{3}\right)$, $\left(\alpha_{i}^{4}, \beta_{i}^{4}\right)$ are respectively coefficients pairs of four found samples. These four samples have different cycles, so a new cycle is interpolated as

$$
T_{d}=a T_{1}+b T_{2}+c T_{3}+d T_{4}
$$

Angular trajectories of all degrees of freedom of the animated locomotion will be synthesized in time domain by Inverse Fourier Transform:

$$
\begin{gathered}
m_{d}(t)=\frac{1}{2} \alpha_{0}^{d}+\sum_{k=1}^{N} \alpha_{k}^{d} \cos \left(\frac{k \pi t}{T_{d}}\right)+\beta_{k}^{d} \sin \left(\frac{k \pi t}{T_{d}}\right) \\
\text { V. RESUlts }
\end{gathered}
$$

\section{A. Reactive locomotions}

The perspective of our work is to animate reactive locomotions. For current stage, we present some off-line reactive ones. Desired global locomotions path were pre-defined, and they include parts of which the velocities or tangential directions are varied. Two examples of such locomotions are presented in figure 12. These two hybrid locomotions can build a scenario including nonholonomic and holonomic locomotions. Such a scenario is an interaction between two persons in a corridor corner (see on the top-left corner in figure 12). Each person performs a hybrid locomotion. The person A goes first along the main corridor, then avoiding the other person B by sideward steps and finally, continue to go ahead. The other walker B goes from the left side of the main corridor on a straight line and turn on his right when arriving in the main corridor. He will meet the person A and will stop to let A pass first, then he will continue.

\section{B. Intentional locomotions}

An intentional trajectory from initial to final configurations is produced by using the inverse optimal control approach in [11]. Such a locomotion is presented in figure 13 (a) and it is close to locomotions acquired from data of human locomotion experiment in the neighboring space [15]. Like in [15], the mannequin adapts the body orientation to the goal during locomotion. We chose desired velocities and then generated the locomotion. A postures sequence of the result during the animation was shown in figure 13 (b).

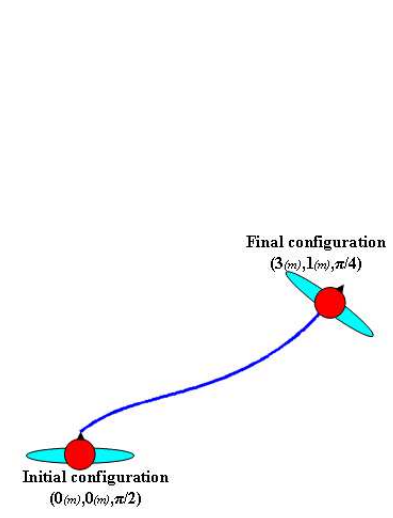

(a)

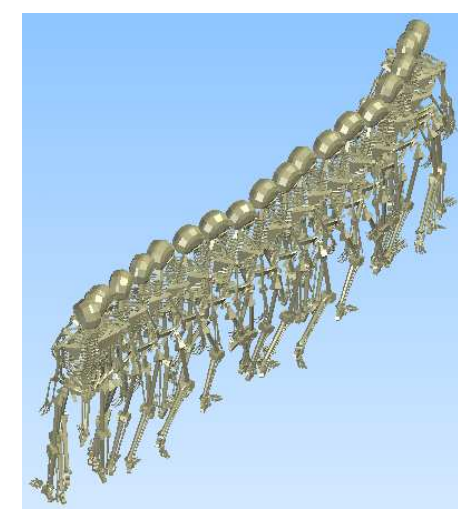

(b)
Fig. 13. The initial and final configurations of an intentional goal walking (a) and the animated locomotion generated by our controller (b)

\section{CONCLUSION}

Nonholonomic and holonomic locomotions have been combined in our locomotion controller. We produced some feasible locomotions (look like human walking). The ongoing work allows us to animate locomotions following predefined paths in some interesting scenarios. Moreover, with the optimizer used in [11], an optimal (human-like) trajectory would be planned and tracked by locomotions generated by our controller. From a top-down approach, we defined a computational model explaining trajectory shape of human locomotion between different configurations. And now, from these different configurations, we could generate "human-like" locomotions. It seems to be an inverse application of top-down approach. Nevertheless, these locomotions were created without any feedback information of equilibrium and contact on the ground. It's just a movement synergy of the root - free flyer object and 

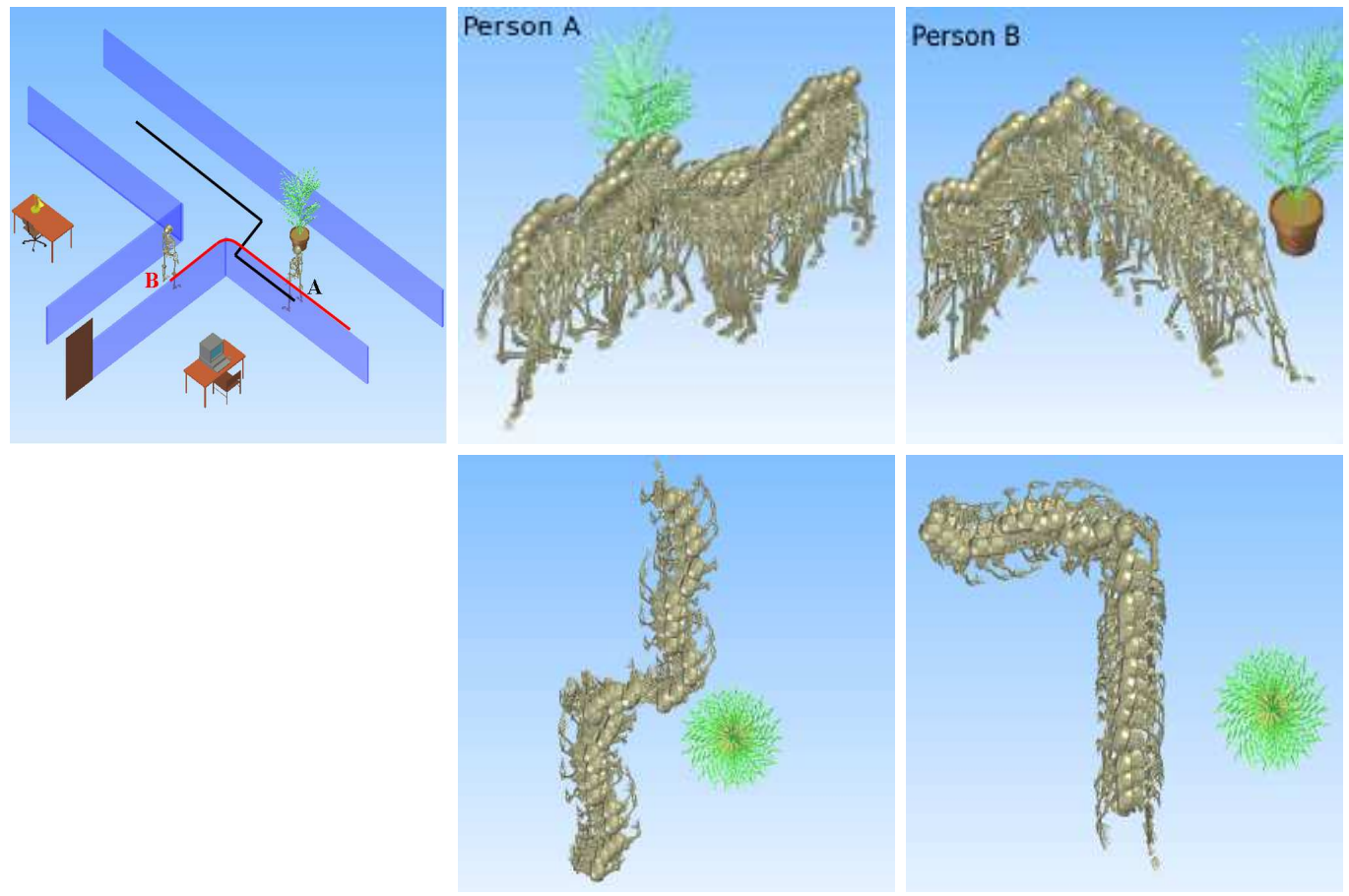

Fig. 12. The interaction scenario at the corridor on the top-left corner. Two persons are on starting points of them. Person A performed a hybrid locomotion of forward walking and sideward steeping: the perspective view and the top view are in the middle of the first line and the second line respectively. Person B performed a hybrid locomotion of forward walking and right turning: the perspective view and the top view are on the right of the first line and the second line respectively.

all joints participating in human locomotion. So, the slide of feet on the ground could not be completely eliminated. We tried to reduce the slide by fixing an impacting foot on the ground during the swing phase of the other one. Synthesized locomotions represent feasible movements and satisfy user's directives. Even when the input velocities change suddenly, reactive animations are simultaneously generated and they look like what humans do. Nevertheless, these inputs have to be in the envelop of control space data structure. In the scope of normal walking, the database in our Motion library could synthesize most of human-like locomotions. For further applications, our objective is to control locomotions by using a joystick and to apply the motion planner in the case with the appearance of obstacles.

\section{ACKNOWLEDGMENT}

This work is financed by French ANR project "Locanthrope". We thank Sebastien Dalibard for his valuable help.

\section{REFERENCES}

[1] F. C. Anderson and M. G. Pandy, "Dynamic optimization of human walking," Journal of Biomechanical Engineering, vol. 123, pp. 381-390, 2001.

[2] K. Bathe, Finite element procedures in engineering analysis. PrenticeHall Englewood Cliffs, NJ, 1982.

[3] A. Berthoz and I. Viaud-Delmon, "Multisensory integration in spatial orientation," Current Opinion in Neurobiology, vol. 9, pp. 708-712, 1999.

[4] A. Carlsen, P. Kennedy, K. Anderson, E. Cressman, P. Nagelkerke, and R. Chua, "Identifying visual-vestibular contributions during targetdirected locomotion," Neuroscience letters, vol. 384, no. 3, pp. 217-221, 2005.

[5] M. Cinelli and W. Warren, "Eyes or head: Which has the greatest effect on steering control?" Journal of Vision, vol. 9, no. 8, pp. 1122-1122, 2009.
[6] H. Hicheur and A. Berthoz, "How do humans turn? Head and body movements for the steering of locomotion," in The 5th International Conference on Humanoid Robots, Tsukuba, Japan, 2005.

[7] H. Hicheur, Q.-C. Pham, G. Arechavaleta, J.-P. Laumond, and A. Berthoz, "The formation of trajectories during goal-oriented locomotion in humans. I. A stereotyped behaviour," In European Journal of Neuroscience, vol. 26, 2007.

[8] O. Khatib, L. Sentis, J. Park, and J. Warren, "Whole body dynamic behavior and control of human-like robots," International Journal of Humanoid Robotics, vol. 1, no. 1, pp. 29-43, 2004.

[9] J.-P. Laumond, S. Sekhavat, and F. Lamiraux, Robot Motion Planning and Control. J.-P. Laumond (Ed.), Lectures Notes in Control and Information Sciences 229, Springer, 1998, ch. Guidelines in Nonholonomic Motion Planning for Mobile Robots.

[10] K. Mombaur, J.-P. Laumond, and I. Yoshida, "An optimal control based formulation to determine natural locomotor paths for humanoid robots," Advanced Robotics, vol. 24, no. 4, 2010.

[11] K. Mombaur, A. Truong, and J.-P. Laumond, "From human to humanoid locomotion - An inverse optimal control approach," Autonomous Robots, vol. 28, no. 3, pp. 369-383, 2010.

[12] J. Pettre and J.-P. Laumond, "A motion capture-based control-space approach for walking mannequins," Computer Animation Virtual Worlds, vol. 17 , no. 2, pp. 109-126, 2006.

[13] M.-H. Raibert, Legged Robots That Balance. MIT Press, Cambridge, 1986.

[14] Saunders, V. T. Inman, and H. D. Eberhart, "The major determinants in normal and pathological gait," Journal of Bone Joint Surgery, vol. 35, no. 3 , pp. 543-558, 1953.

[15] T.-V.-A. Truong, K. Mombaur, and J.-P. Laumond, "On the relationship between body orientation and direction of motion during human locomotion," in 19th Symposium of International Society for Posture and Gait Research, Bologna, Italy, 2009.

[16] M. Unuma, K. Anjyo, and R. Takeuchi, "Fourier principles for emotionbased human figure animation," in SIGGRAPH '95: Proceedings of the 22nd annual conference on Computer graphics and interactive techniques. New York, USA: ACM, 1995, pp. 91-96.

[17] M. Vukobratovic and B. Borovac, "Zero-moment point - thirty five years of its life," International Journal of Humanoid Robotics, vol. 1, no. 1, pp. 157-173, 2004.

[18] www.cgal.org, "CGAL, computational geometry algorithms library." 